\title{
Transverse Spin Structure Function $g_{2}\left(x, Q^{2}\right)$ in the Valon
} model

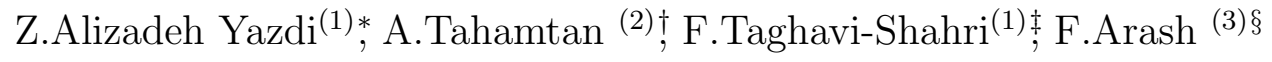 \\ and M.E.Zomorrodian (1) \\ (1) Department of Physics, Ferdowsi University of Mashhad, P.O. Box 1436, \\ Mashhad, Iran \\ (2) Department of Physics, University of Tehran, North Kargar Avenue, \\ Tehran 14395-547, Iran \\ (3) Physics Department, Tafresh University, Tafresh, Iran
}

October 30, 2018

\begin{abstract}
The spin dependent structure function, $g_{2}^{w w}$, is calculated in the valon model. A simple approach is given for the determination of the twist-3 part of the $\overline{g_{2}}\left(x, Q^{2}\right)$ in Mellin space; thus, enabling us to obtain the full transverse structure function, $g_{2}\left(x, Q^{2}\right)$ for proton, neutron and the deuteron. In light of the new data, we have further calculated the transversely polarized structure function of $g_{2}^{3 \mathrm{He}}\left(x, Q^{2}\right)$. Our results are checked against the experimental data and nice agreements are observed.
\end{abstract}

*zahra.alizadehyazdi@stu.um.ac.ir

†azamtahamtan2@yahoo.com

‡taghavishahri@um.ac.ir

§farash@cic.aut.ac.ir

『 zomorrod@ferdowsi.um.ac.ir 


\section{INTRODUCTION}

The nucleon polarized structure functions $g_{1,2}\left(x, Q^{2}\right)$ are important tools in understanding the nucleon substructure. In particular, they are indispensable elements for the understanding of the spin dependent parton distributions and their correlations. The $g_{2}\left(x, Q^{2}\right)$ structure function is important because it probes transversely and also longitudinally polarized parton distributions inside the nucleon. $g_{2}\left(x, Q^{2}\right)$ structure function is also sensitive to the higher twist effects, such as quark gluon correlations. They do not disappear even at large $Q^{2}$ values and not easily interpreted in pQCD $[1,2]$. Since $g_{2}\left(x, Q^{2}\right)$ is the only function related to the quark-gluon interaction, learning about its behavior will render further insight into the spin structure of the nucleon beyond the simple quark parton model.

Thus, the main purpose of this paper is to calculate transverse spin structure function, $g_{2}\left(x, Q^{2}\right)$. As such, it requires considering both the twist-2 and the twist-3 contributions. Here we will present a simple method to extract twist-3 part. The twist-2 part is well understood, and requires knowledge about the $g_{1}\left(x, Q^{2}\right)$ structure function. Therefore, first we will briefly review $g_{1}\left(x, Q^{2}\right)$ in the context of the so called valon model representation of hadrons.

Finally, the outcome of our results is checked against the experimental data from $[3,4,5$, $6,7]$, and compared with other phenomenological models.

The lay out of the paper is as follows: In section 2, we briefly present a review of the polarized nucleon structure function in the valon model. Section 3 deals with the calculation of $g_{2}\left(x, Q^{2}\right)$ spin structure function and discusses the numerical results. We also provide some discussion on the effect of higher twists. Section 4 is devoted to the sum rules. Our conclusions are given in section 5 . 


\section{A brief review of spin structure functions in the valon model}

The valon model is a phenomenological model, originally proposed by R. C. Hwa, [8] in early 80 's to provide a bridge between the naive quark model and the partonic structure of the hadrons. The model had many successes. It was improved later by Hwa [9] and Others $[10,11,12,13,14,15]$. It was further extended to include the polarized cases $[16,17,18,19]$. The model views a hadron as three (two) constituent quark like objects called valons. Each valon is defined to be a dressed valence quark with its own cloud of sea quarks and gluons. The dressing processes are described by QCD. At high enough $Q^{2}$ values the structure of a valon can be resolved, but At low $Q^{2}$ values, the internal structure of the valon cannot be resolved and it behave as a constituent quarks of the hadron.

In valon model the polarized parton distributions of a polarized hadron are given by the following convolution integral :

$$
\delta q_{i}^{h}\left(x, Q^{2}\right)=\sum \int_{x}^{1} \frac{d y}{y} \delta G_{\text {valon }}^{h}(y) \delta q_{i}^{\text {valon }}\left(\frac{x}{y}, Q^{2}\right)
$$

where $\delta G_{\text {valon }}^{h}(y)$ is the valon helicity distribution in the hosting hadron; that is, it is the probability of finding a polarized valon inside the polarized hadron. In the Next-toLeading order, $\delta G_{\text {valon }}^{h}(y)$ marginally depends on $Q^{2}$. These distributions are shown in (Fig.1).

The term $\delta q_{i}^{\text {valon }}\left(x / y, Q^{2}\right)$ in Eq.(1) is the polarized parton distribution (PPDFs) inside a valon and are obtained from the solutions of DGLAP evolution equations in the valon. Now, using the convolution integral, one can obtain the polarized hadron structure functions as follows:

$$
g_{1}^{h}\left(x, Q^{2}\right)=\sum_{\text {valon }} \int_{x}^{1} \frac{d y}{y} \delta G_{\text {valon }}^{h}(y) g_{1}^{\text {valon }}\left(\frac{x}{y}, Q^{2}\right)
$$




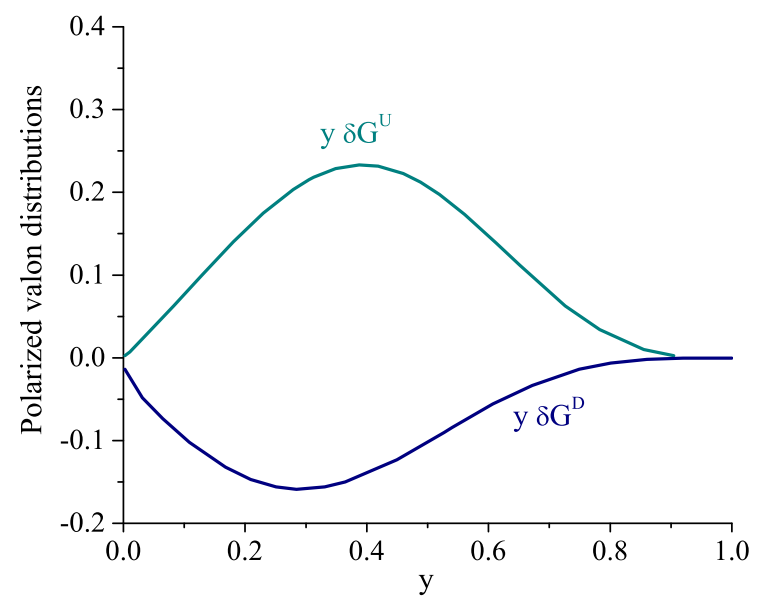

Figure 1: (Color online) polarized valon distribution functions for $\mathrm{U}$ and $\mathrm{D}$ valon types (the helicity distributions for the valons) inside the proton.

where $g_{1}^{\text {valon }}\left(\frac{x}{y}, Q^{2}\right)$ is the polarized structure function of the valon. The details of actual calculations are given in $[16,18]$. In short, the following two steps lead us to both the polarized PPDFs and polarized nucleon structure functions:

- Calculate the PPDFs in the valon using DGLAP equations;

- With a phenomenological approach, the helicity distributions of the valons in a nucleon is obtained. then, they are used in Eqs.(1) and (2)to get the polarized parton distribution functions (PPDFs) and the polarized nucleon structure up to $Q^{2}=10^{7} \mathrm{GeV}^{2}$

It should be noted that the valon model is only a phenomenological model. As such, initial conditions, as inputs to the DGLAP equations, are chosen based on phenomenological arguments. The results obtained for the proton structure function, $g_{1}^{p}\left(x, Q^{2}\right)$ from this model are in excellent agreement with all available experimental data [20, 21, 22, 23, 24, 25]. In Fig.2 we only present a sample of the results along with the existing data. The parton distributions so obtained will be used here to calculate $g_{2}\left(x, Q^{2}\right)$. 

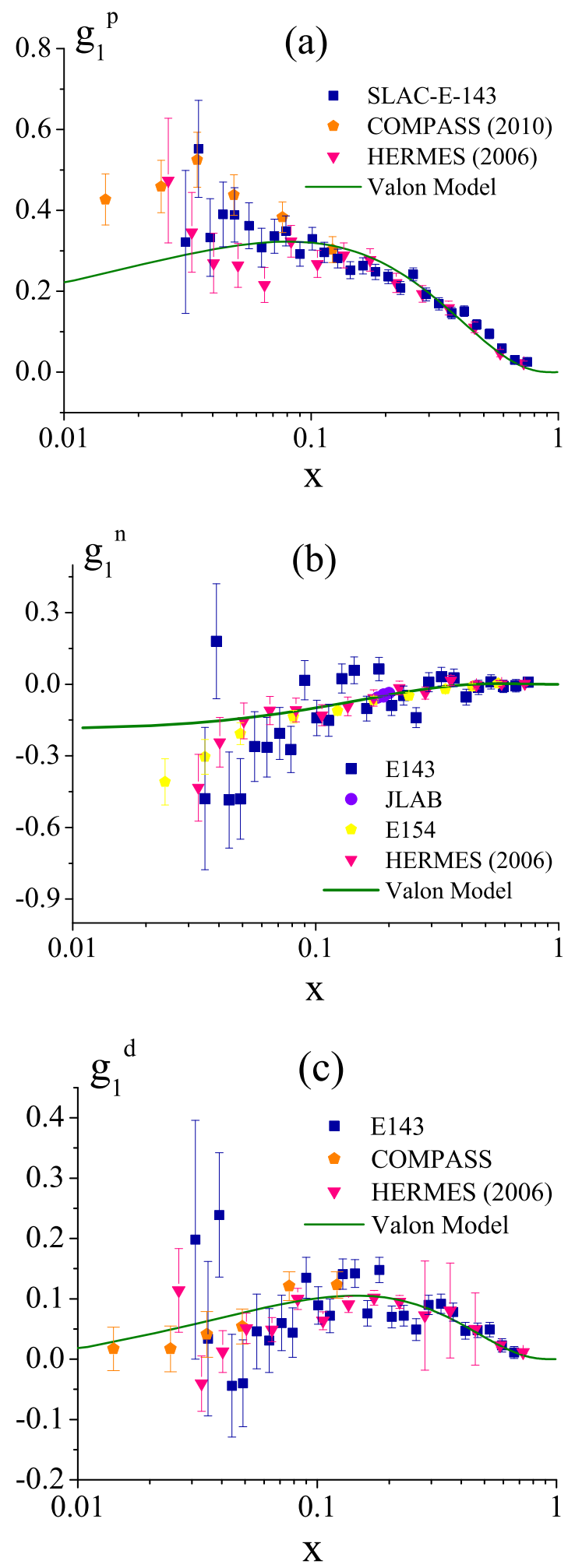

Figure 2: (Color online) Polarized proton, neutron and deuteron structure function $g_{1}\left(x, Q^{2}\right)$ at $Q^{2}=$ $5 \mathrm{GeV}^{2}$. The results from valon model are compared with the experimental data [20, 21, 22, 23, 24, 25]. 


\section{Transverse spin-dependent structure function $g_{2}\left(x, Q^{2}\right)$}

Polarized Deep Inelastic Scattering (DIS) mediated by a photon exchange, probes two spin structure functions: $g_{1}\left(x, Q^{2}\right)$ and $g_{2}\left(x, Q^{2}\right)$. If the target is transversely polarized, the total cross section is a combination of these two structure functions. Transverse spin structure function, $g_{2}\left(x, Q^{2}\right)$, is made up of two components: a twist-2 part, $g_{2}^{w w}$, and a mixed twist part, $\bar{g}_{2}\left(x, Q^{2}\right)$. Therefore, it can be written as [26]:

$$
g_{2}\left(x, Q^{2}\right)=g_{2}^{w w}\left(x, Q^{2}\right)+\bar{g}_{2}\left(x, Q^{2}\right)
$$

where

$$
\bar{g}_{2}\left(x, Q^{2}\right)=-\int_{x}^{1} \frac{\partial}{\partial y}\left(\frac{m}{M} h_{T}\left(y, Q^{2}\right)+\xi\left(y, Q^{2}\right)\right) \frac{d y}{y} .
$$

The twist-2 part, $g_{2}^{w w}$, comes from OPE. The $\bar{g}_{2}\left(x, Q^{2}\right)$ receives a contribution from the transversely polarized quark distributions $h_{T}\left(x, Q^{2}\right)$ plus a contribution that comes from a twist-3 component, an indication of $q g q$ correlations, given by $\xi\left(y, Q^{2}\right)$ term in Eq.(4). These higher twist corrections arise from the non-perturbative multi parton interactions. Their contributions at low energy increase as $\frac{1}{Q^{\tau}}$, reflecting the confinement. Any non-zero result for this term at a given $Q^{2}$ will reflect a departure from the non-interacting partonic regime [27].

$g_{2}^{w w}$ is related to the $g_{1}$ structure function by the Wandzura-Wilczek relation [28] as follows,

$$
g_{2}^{w w}\left(x, Q^{2}\right)=-g_{1}\left(x, Q^{2}\right)+\int_{x}^{1} g_{1}\left(y, Q^{2}\right) \frac{d y}{y} .
$$

\subsection{Calculation of the twist-2 term, $g_{2}^{w w}\left(x, Q^{2}\right)$}

We begin with Eq.(5). Since $g_{1}\left(x, Q^{2}\right)$ is known in the valon model [16, 18], we utilize them without any additional free parameter and evaluate the twist-2 part of $g_{2}\left(x, Q^{2}\right)$; namely $g_{2}^{w w}\left(x, Q^{2}\right)$, according to the Eq.(5). The results are shown in Fig. 3 for proton. We have also included the findings of $[29,30]$ for the purpose of comparison. 


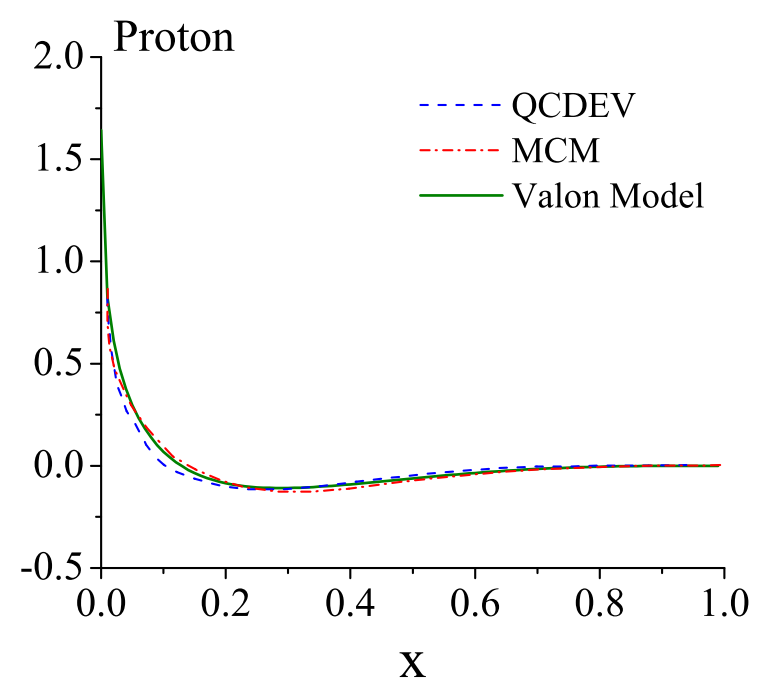

Figure 3: (Color online) Transverse polarized proton structure functions, $g_{2}^{w w}\left(x, Q^{2}\right)$ at $Q^{2}=5 G e V^{2}$. We compare our results with the results from other phenomenological models $[29,30]$.

\subsection{Calculating the twist-3 term, $\bar{g}_{2}\left(x, Q^{2}\right)$}

As mentioned, the function $\bar{g}_{2}\left(x, Q^{2}\right)$ has two terms. The first term is a twist-2 contribution related to the transverse polarization of quarks in the nucleon. It is suppressed by the quark to nucleon mass ratio and will be ignored here due to its negligibility. The second part is a twist-3 contribution, reflecting the quark-gluon correlations. In the following we will focus on this part.

In large $N_{c}$ limit, Ali, Braun and Hiller found that the $Q^{2}$-evolution of $\bar{g}_{2}\left(x, Q^{2}\right)$ is qualified by simple DGLAP type equation with awhile difference between the anomalous dimensions and the twist-2 distribution[31, 32]. It implies that $\bar{g}_{2}\left(x, Q^{2}\right)$ obeys the following simple equation :

$$
\bar{g}_{2}\left(n, Q^{2}\right)=L^{\frac{\gamma_{n}^{g}}{2 b_{0}}} \bar{g}_{2}\left(n, Q_{0}^{2}\right)
$$

where,

$$
\begin{gathered}
\bar{g}_{2}\left(n, Q^{2}\right)=\int_{0}^{1} x^{n-1} \bar{g}_{2}\left(x, Q^{2}\right) d x, \\
L \equiv \frac{\alpha_{s}\left(Q^{2}\right)}{\alpha_{s}\left(Q_{0}^{2}\right)},
\end{gathered}
$$


and

$$
\begin{array}{r}
b_{0}=\frac{11}{3} N_{c}-\frac{2}{3} N_{f} \\
\gamma_{n}^{g}=2 N_{c}\left(S_{n-1}-\frac{1}{4}+\frac{1}{2 n}\right) \\
S_{n-1}=\sum \frac{1}{j}
\end{array}
$$

$\alpha_{s}$ is the strong coupling constant, $N_{f}$ is the number of flavor and $S_{n-1}$ are the Harmonic functions. Our purpose is to find the $Q^{2}$-evolution of $\bar{g}_{2}$ with some appropriate initial conditions in moment space. Then we can make a transformation to the momentum space and evaluate the twist-3 contribution to the transverse spin structure function. This is done in two steps, as is the case in the valon model. The first step involves finding a solution to Eq.(6) in a valon. The second step is to convolute the results obtained in the first step with the valon distribution in the nucleon. This will give the nucleon structure function.

We take $Q_{0}^{2}=0.238$ as our initial scale which is also used in our original calculations of various parton distributions. This value of $Q_{0}^{2}$ corresponds to a distance scale of $0.36 \mathrm{fm}$ which is roughly equal to or less than the radius of a valon[11]. The initial input function for $\bar{g}_{2}^{\text {valon }}\left(z, Q_{0}^{2}\right)$ is (See the Appendix A) :

$$
\bar{g}_{2}^{\text {valon }}\left(z, Q_{0}^{2}\right)=A \delta(z-1)
$$

The justification for this choice is as following: In the momentum space one can write

$$
\bar{g}_{2}^{\text {valon }}\left(z, Q^{2}\right)=f\left(Q^{2}\right) \bar{g}_{2}\left(z, Q_{0}^{2}\right)=f\left(Q^{2}\right) A \delta(z-1)
$$

Note that for $Q^{2}=Q_{0}^{2}$ we get $f\left(Q^{2}\right) \rightarrow f\left(Q_{0}^{2}\right)=1$ which is apparent from the definition of $L$ in Eq.(8), thus, arriving at Eq.(12). This simple choice for the initial input in $\bar{g}_{2}^{\text {valon }}\left(z, Q_{0}^{2}\right)$ stems from the knowledge that it is related to the quark gluon correlations, which in turn, is related to the Green function in the momentum space. In the momentum space the correlation function is composed of a Dirac Delta term and a function that is 
related to the momentum. consequently, at the initial $Q_{0}^{2}$ we can simply assume that $\bar{g}_{2}^{\text {valon }}\left(z, Q_{0}^{2}\right)$ is proportional to Dirac Delta function which emphasizes the conservation of energy- momentum and the fact that at such a low $Q_{0}^{2}$ a valon behaves as an object without any internal structure. The last point is built in the definition of a valon. So, in the moment space the Delta function becomes unity and we can write

$$
\bar{g}_{2}\left(n, Q_{0}^{2}\right)=A \times 1
$$

all of the QCD effects are summarized in $A$. This coefficient will be extracted from the experimental data. A fit to E143 data yeilds a value 0.01 for A. Having specified the initial input values, the moments of $\bar{g}_{2}^{\text {valon }}\left(z, Q^{2}\right)$ in a valon are readily obtained, with the aid of Eq.(14). An inverse Mellin transformation then takes us to the momentum space, giving $\bar{g}_{2}^{\text {valon }}\left(z, Q^{2}\right)$ structure function. For example at $Q^{2}=10 G e V^{2}$, we have:

$$
\bar{g}_{2}^{\text {valon }}\left(z, Q^{2}=10 G e V^{2}\right)=0.028 z^{4.645}
$$

This completes the first step described above. In Fig. $4, \bar{g}_{2}^{\text {valon }}\left(z, Q^{2}\right)$ is shown for different values of $Q^{2}$. According to Fig.5 as our calculations for the values of $N_{c} \geq 100$ leads to a similar distribution for $\bar{g}_{2}^{\text {valon }}\left(z, Q^{2}\right)$ for the whole range of $z$, We are choosing the optimal value of $N_{c}$ which is equal to 100 (reminder that Eq. (6) is valid only for large $N_{C}$ ).

The second step involves the convolution process which takes us to the hadronic level. This is similar to the earlier procedure that we have used to extract $g_{1}^{p, n}\left(x, Q^{2}\right)$. Similar to $g_{1}$, we can write:

$$
g_{2}^{h}\left(x, Q^{2}\right)=\sum_{\text {valon }} \int_{x}^{1} \frac{d y}{y} \Delta_{T} G_{\text {valon }}^{p}(y) g_{2}^{\text {valon }}\left(\frac{x}{y}, Q^{2}\right)
$$

where $\Delta_{T} G_{v a l o n}^{p}(y)$ is the transverse valon distribution functions describing the probability of finding a valon with spin aligned or anti-aligned with the transversly polarized proton. In fact, $\Delta_{T} G_{\text {valon }}^{p}(y)$ is identical to $\delta G_{\text {valon }}^{p}(y)$ in the longitudinal case. This is so, because we know that in the non-relativistic limit of the quark motion, the PPDFs and transversity 


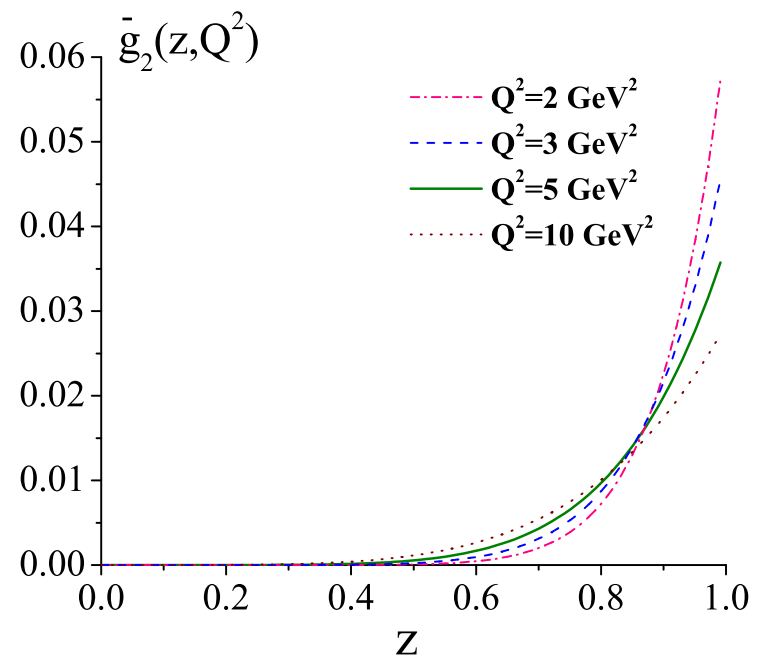

Figure 4: (Color online) The twist-3 part of proton transverse spin structure function $\bar{g}_{2}\left(z, Q^{2}\right)$ in the valon, at $Q^{2}=2,3,5,10 \mathrm{GeV}^{2}$.

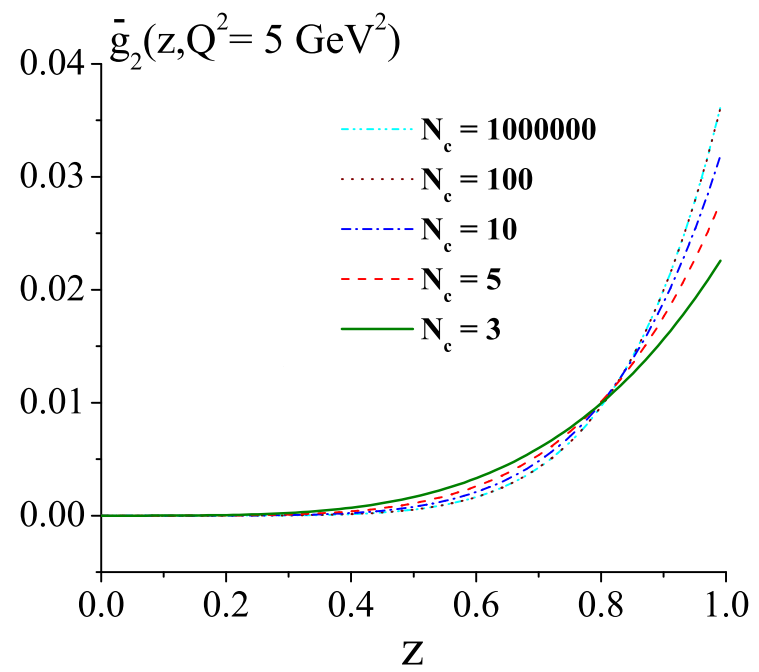

Figure 5: (Color online) The twist-3 part of proton transverse spin structure function $\bar{g}_{2}\left(z, Q^{2}\right)$ in the valon at $Q^{2}=5 \mathrm{GeV}^{2}$ for different $N_{c}$. 
distribution would be identical, since the rotations and Euclidean boosts commute and a series of boosts and rotation can convert a longitudinal polarized proton into a transversely polarized one with an infinite momentum [33, 34, 35]. Finally substituting the valon helicity distribution, $\delta G^{U(D)}(y)$ in the corresponding hadron leads us to $\bar{g}_{2}\left(x, Q^{2}\right)$. In Fig.6 we present $x^{2} \bar{g}_{2}\left(x, Q^{2}\right)$ for both proton and deuteron along with the E143 data [3] . We have compared our results with those obtained in the Bag model [29] and that of Wakamatsu [32].

Finally, adding $\bar{g}_{2}\left(x, Q^{2}\right)$ and $g_{2}^{w w}\left(x, Q^{2}\right)$, gives the full $g_{2}\left(x, Q^{2}\right)$. The final results for
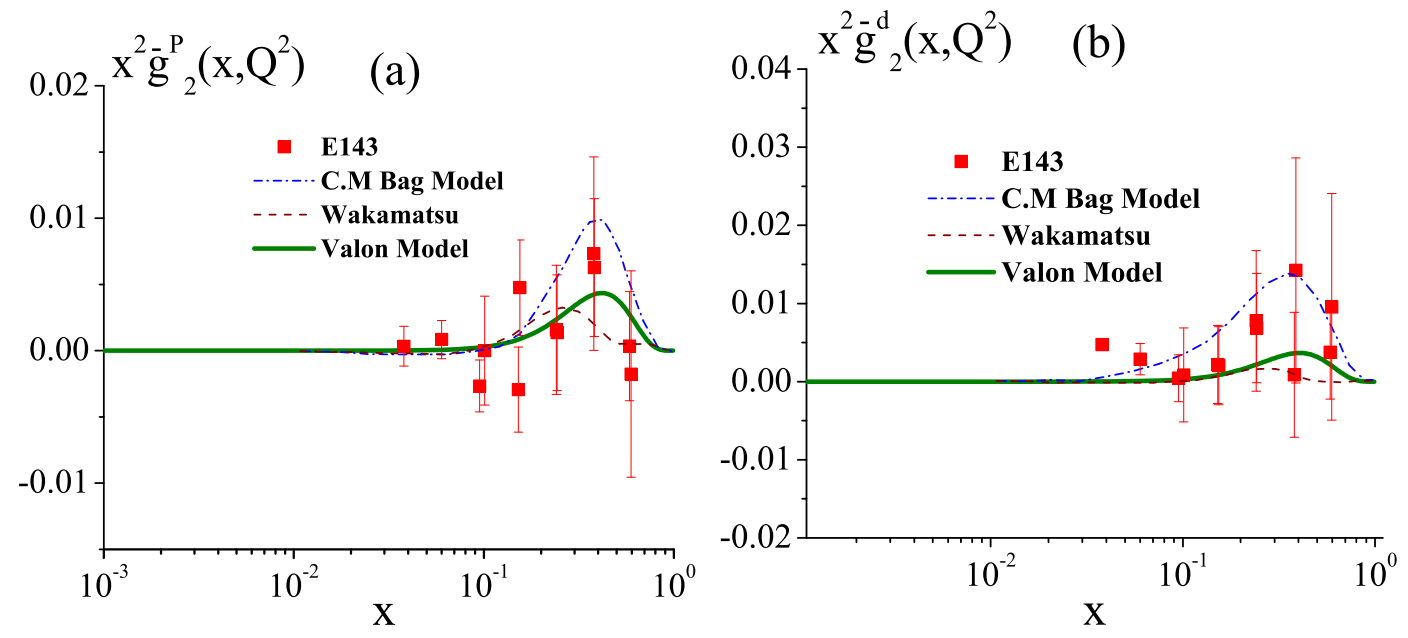

Figure 6: (Color online) The twist-3 part of proton and deuteron transverse spin structure function, $x^{2} \bar{g}_{2}\left(x, Q^{2}\right)$ at $Q^{2}=5 \mathrm{GeV}^{2}$. we compared our results with E143 data [3] and other phenomenological groups [29], [32].

$x g_{2}\left(x, Q^{2}\right)$ are presented in Fig.7 for proton, neutron and deuteron at different values of $Q^{2}$. Confronting with the experimental data, in Fig.8 we show our results for the full transversely polarized structure function $g_{2}\left(x, Q^{2}\right)$ for proton, neutron and deuteron and the experimental findings of $[3,4,6]$. 

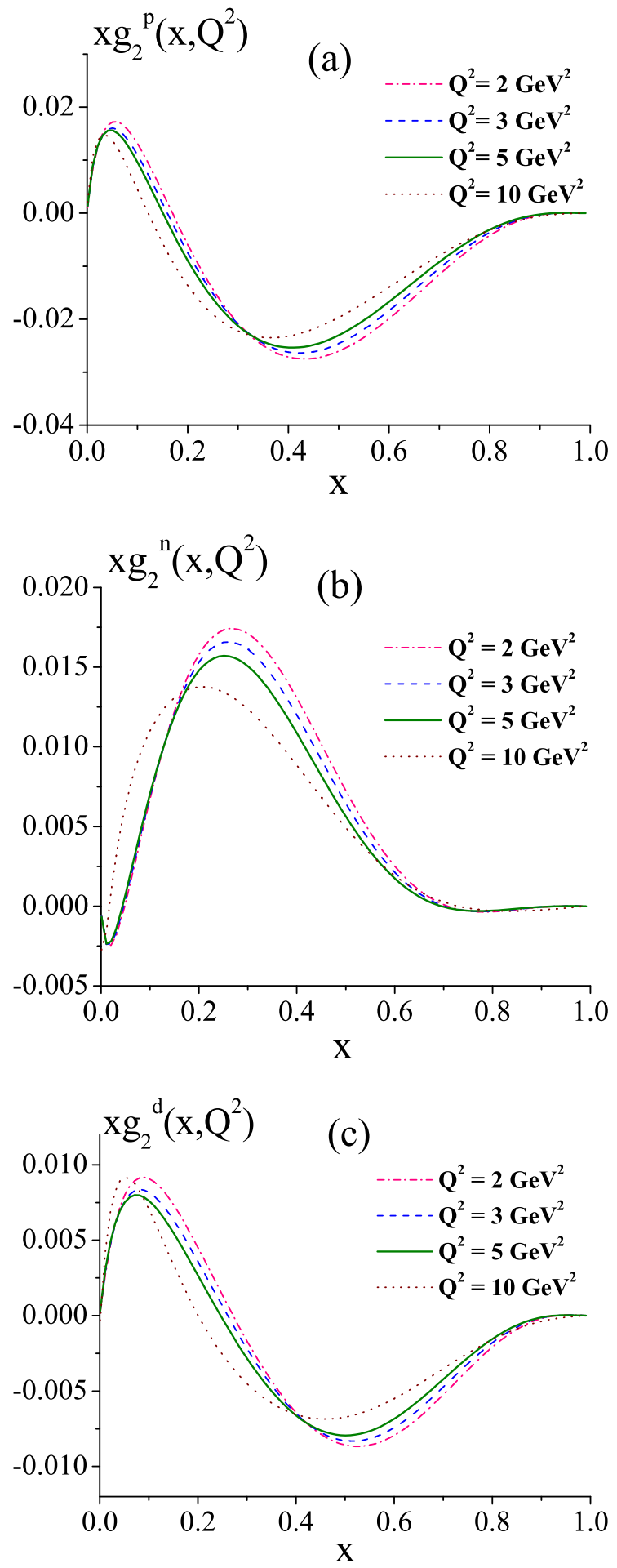

Figure 7: (Color online) $x g_{2}\left(x, Q^{2}\right)$ proton, neutron and deuteron at $Q^{2}=2,3,5,10 \mathrm{GeV}^{2}$. 

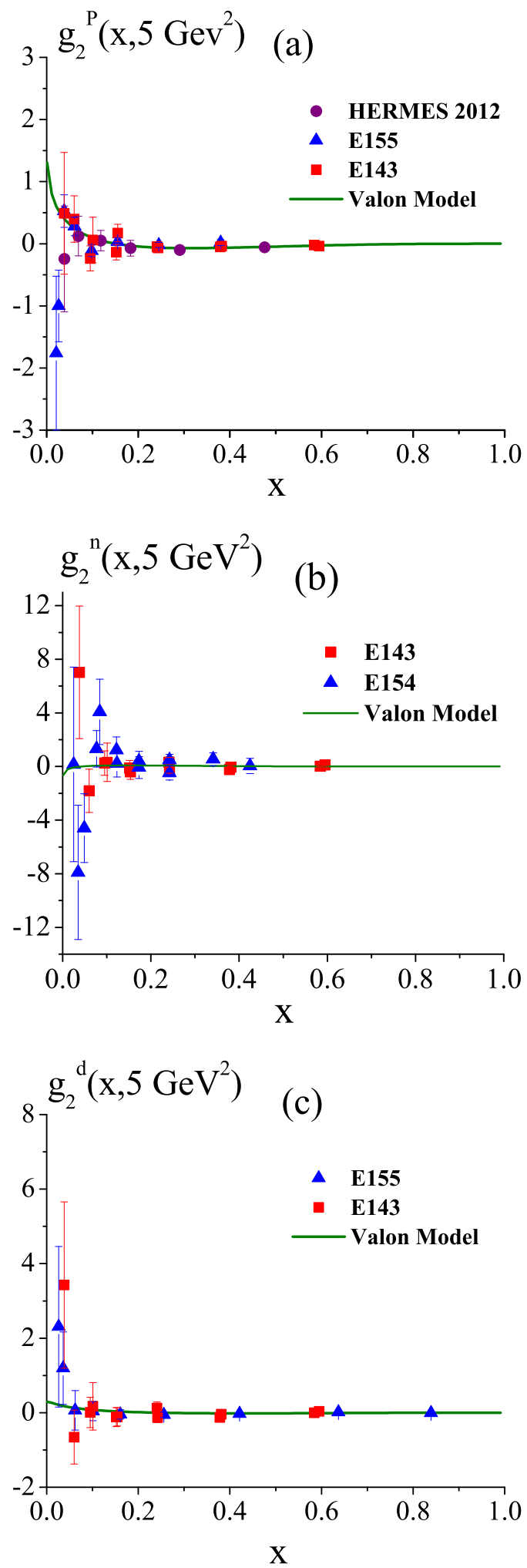

Figure 8: (Color online) Full transverse polarized structure function $g_{2}\left(x, Q^{2}\right)$ for proton, neutron and deuteron at $Q^{2}=5 \mathrm{GeV}^{2}$ from valon model and it is compared with experimental data $[3,4,6]$. 


\subsection{The case of $g_{2}^{3 H e}\left(x, Q^{2}\right)$}

It is intriguing to investigate the $g_{2}^{3_{\mathrm{He}}}\left(x, Q^{2}\right)$ as a special case, since there are some newly released data on $g_{2}^{3 \mathrm{He}}\left(x, Q^{2}\right)$, and the conformity of our result with the experiment will lend further justification to the approach adopted here.

The $g_{2}^{3^{H} \mathrm{He}}\left(x, Q^{2}\right)$ structure function can be viewed as the sum of $g_{2}^{n}\left(x, Q^{2}\right)$ and $g_{2}^{p}\left(x, Q^{2}\right)$, each convoluted with the spin dependent nucleon light-cone momentum distributions, $\Delta f_{3_{H e}}^{N}(y)$, where $y$ is the ration of " + components of the light cone momenta of struck nucleon to nucleus. One will have

$$
g_{2}^{3_{H e}}\left(x, Q^{2}\right)=\int_{x}^{3} \frac{d y}{y} \Delta f_{3_{H e}}^{n}(y) g_{2}^{n}\left(x / y, Q^{2}\right)+2 \int_{x}^{3} \frac{d y}{y} \Delta f_{3_{H e}}^{p}(y) g_{2}^{p}\left(x / y, Q^{2}\right)
$$

So, using Eq.(17), this should be straightforward. All is needed are two functions, namely, $\Delta f_{3_{H e}}^{p}(y)$ and $\Delta f_{3_{H e}}^{n}(y)$. They can be extracted from the numerical results of [36, 37]. In Fig. 9 we have plotted $g_{2}^{3}{ }^{H e}\left(x, Q^{2}\right)$ together with the experimental data from [38, 39, 40]. As can be seen from the figure, our approach is in fair agreement with the experimental

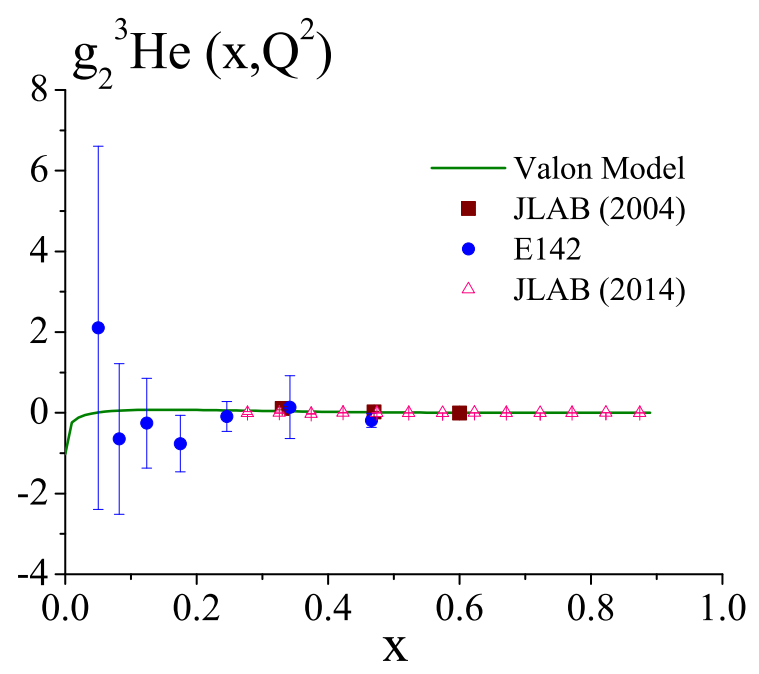

Figure 9: (Color online) Full transversely polarized $3_{\mathrm{He}}$ structure function, $g_{2}\left(x, Q^{2}\right)$ at $Q^{2}=5 \mathrm{GeV}^{2}$. The data points are from $[38,39,40]$.

data. 


\section{The Sum Rules}

There are two important and well known sum rules regarding $g_{1}\left(x, Q^{2}\right)$ and $g_{2}\left(x, Q^{2}\right)$. The first one is OPE sum rule:

$$
\begin{gathered}
\Gamma_{1}^{n}=\int_{0}^{1} x^{n} g_{1}\left(x, Q^{2}\right) d x=\frac{a_{n}}{2}, n=0,2,4, \ldots \\
\Gamma_{2}^{n}=\int_{0}^{1} x^{n} g_{2}\left(x, Q^{2}\right) d x=\frac{1}{2} \frac{n}{n+1}\left(d_{n}-a_{n}\right), n=2,4, \ldots
\end{gathered}
$$

where $a_{n}$ and $d_{n}$ are the twist-2 and the twist-3 matrix element operators, respectively. The study of these sum rules is easy for simplest case $(n=2)$ where the twist three effects are exist $[1,2]$.

The second one is the Burkhardt- Cottingham sum rule [41]. It states that the first moment of $g_{2}\left(x, Q^{2}\right)$ structure function vanished.

$$
\int_{0}^{1} g_{2}\left(x, Q^{2}\right) d x=0
$$

Since $g_{2}=g_{2}^{w w}+\bar{g}_{2}$, upon combining Eq. (18) and Eq. (19) with Eq. (5) provides a third sum rule. They are listed bellow:

$$
\begin{array}{r}
\int_{0}^{1} g_{2}^{w w}\left(x, Q^{2}\right) d x=0 \\
\int_{0}^{1} x^{2} g_{2}^{w w}\left(x, Q^{2}\right) d x=-\frac{1}{3} a_{2} \\
\int_{0}^{1} x^{2} \bar{g}_{2}\left(x, Q^{2}\right) d x=\frac{1}{3} d_{2}
\end{array}
$$

we have evaluated $d_{2}^{p}, d_{2}^{n}$ in the valon model for a number of $Q^{2}$ values, the results are shown in Fig. 10 and compared with the available data, the bag model, and the QCD Sum Rule results. Table 3 shows our results for the Burkhardt-Cottingham sum rule in the region $0.023<x<0.9$ at $Q^{2}=5 G e v^{2}$. They are checked against the data from HERMES in the same region and also with the findings of E143 and E155 in the range $0.02<x<0.8$. For the purpose of comparison, results from other sources are also included. While $d_{2}^{p}$ is in excellent agreement with the experiment, $d_{2}^{n}$ is less so. However, we also notice that there are fewer data for $d_{2}^{n}$ and thus, making it difficult to arrive in a firm conclusion. 


\begin{tabular}{|c|c|c|c|}
\hline & $a_{2}^{p}$ & $a_{2}^{n}$ & $a_{2}^{d}$ \\
\hline \hline Valon model & 0.01956 & -0.00004 & 0.00874 \\
\hline Lattice QCD [45] & $(3 \pm 0.64) \times 10^{-2}$ & $-(2.4 \pm 4.0) \times 10^{-3}$ & $(13.8 \pm 5.2) \times 10^{-3}$ \\
\hline CM bag model by Song [29] & 0.0210 & $-1.8 \times 10^{-3}$ & 0.0087 \\
\hline E143 [3] & $(2.42 \pm 0.20) \times 10^{-2}$ & - & $(8.0 \pm 0.16) \times 10^{-3}$ \\
\hline
\end{tabular}

Table 1: The twist-2 matrix elemet operators, $a_{2}$, for the proton, neutron and the deuteron, calculated in the valon model. Also included the experimental data and the results from other theoretical investigations.

\begin{tabular}{|c|c|c|c|}
\hline & $d_{2}^{p}$ & $d_{2}^{n}$ & $d_{2}^{d}$ \\
\hline \hline Valon model & 0.00519 & 0.0042 & 0.00437 \\
\hline MIT bag model [29, 42] & 0.01 & 0 & 0.005 \\
\hline QCD sum rule [43] & $-(0.6 \pm 0.3) \times 10^{-2}$ & $-(30 \pm 10) \times 10^{-3}$ & -0.017 \\
\hline QCD sum rule [44] & $-(0.3 \pm 0.3) \times 10^{-2}$ & $-(25 \pm 10) \times 10^{-3}$ & -0.013 \\
\hline Lattice QCD [45] & $-(4.8 \pm 0.5) \times 10^{-2}$ & $-(3.9 \pm 2.7) \times 10^{-3}$ & -0.022 \\
\hline CM bag model by Song [29] & 0.0174 & $-2.53 \times 10^{-3}$ & 0.0067 \\
\hline E143 [3] & $(0.54 \pm 0.5) \times 10^{-2}$ & - & $(3.9 \pm 9.2) 10^{-3}$ \\
\hline
\end{tabular}

Table 2: The twist-3 matrix elemet operators, $d_{2}$, for the proton, neutron and the deuteron, calculated in the valon model. Also included the experimental data and the results from other theoretical investigations.

\begin{tabular}{|c|c|c|c|c|c|}
\hline & bag model by Song $[29]$ & E143 & E155 & HERMES 2012 & Valon model \\
\hline \hline $\int_{0.02}^{0.85} g_{2}^{p}\left(x, Q^{2}\right) d x$ & -0.0016 & $-0.013 \pm 0.028$ & $-0.022 \pm 0.071$ & $0.006 \pm 0.024 \pm 0.017$ & -0.0016 \\
\hline $\int_{0.02}^{0.85} g_{2}^{d}\left(x, Q^{2}\right) d x$ & -0.00287 & - & $0.023 \pm 0.044$ & - & 0.0092 \\
\hline
\end{tabular}

Table 3: The results for the Burkhardt-Cottingham sum rule. 

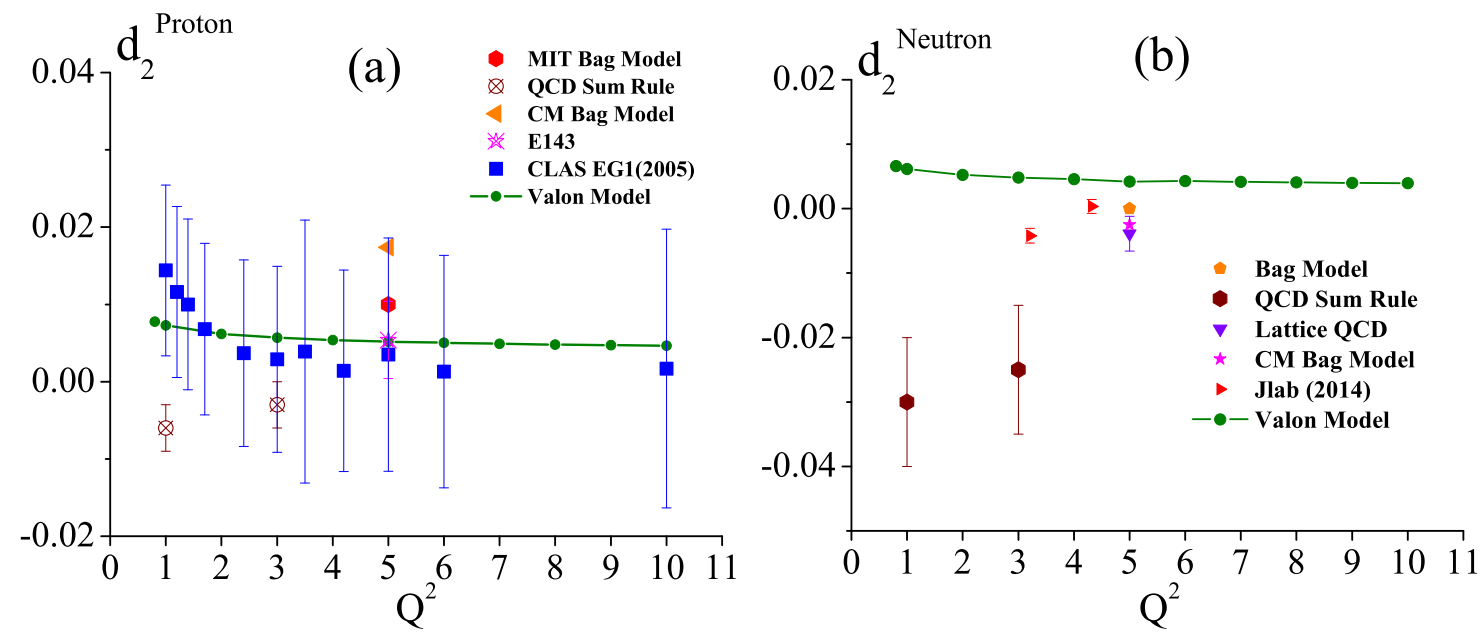

Figure 10: (Color online) $d_{2}^{p}$ and $d_{2}^{n}$ are calculated in valon model. Our results are compared with experimental data as well as with obtained from the results of other phenomenological groups, [3, 29, 40, $42,43,44,45,46]$.

\section{Conclusion}

We have used the so-called valon model to calculate the transverse spin structure functions of the nucleon and the deuteron. To do so, we provide a simple approach for calculating the twist-3 part of the transverse spin structure function $\overline{g_{2}}\left(x, Q^{2)}\right.$ in Mellin space. Furthermore, as a separate check on the validity of our approach, we have considered $g_{2}^{3 H e}\left(x, Q^{2}\right)$ , where we have utilized some light cone momentum distribution and compared with the new data from [40]. Evidently our findings are in agreement with the experiment, rendering the conclusion that hadronic structure functions, both polarized and unpolarized, are nicely described in the valon representation.

\section{Appendix}

Here we attempt to justify our choice of initial input value in $g_{2}^{\text {valon }}\left(x, Q_{0}^{2}\right)$ :

$$
\bar{g}_{2}^{\text {valon }}\left(z, Q_{0}^{2}\right)=A \delta(z-1)
$$

As we know the $g_{2}\left(x, Q^{2}\right)$ is related to quark-gluon-quark correlation. Since, by defi- 
nition, at initial scale, $Q_{0}^{2}$, the valon behaves as an object with no internal structure, it is reasonable to assume that, at such initial scale this object is related to the quark-quark correlations(two-point green function), because at such a low $Q_{0}^{2}$ gluons carry have very small and negligible momentum.

The general form of two-point green function in momentum space is given here (Eq. (2.102) in $[47])$ :

$$
\begin{aligned}
G^{(n)}\left(x_{1}, x_{2}, \cdots, x_{n}\right) \cong & \frac{1}{2}\left(\frac{-i \lambda}{\hbar}\right) \prod_{i=1}^{4} \hbar \frac{i}{p_{i}^{2}-m^{2}+i \epsilon} \int \frac{d^{4} k_{1}}{(2 \pi)^{4}} \frac{d^{4} k_{2}}{(2 \pi)^{4}}(2 \pi)^{4} \delta\left(p_{1}+p_{2}+k_{1}+k_{2}\right) \\
& (2 \pi)^{4} \delta\left(p_{3}+p_{4}-k_{1}-k_{2}\right) \hbar \frac{i}{k_{1}^{2}-m^{2}+i \epsilon} \hbar \frac{i}{k_{2}^{2}-m^{2}+i \epsilon}
\end{aligned}
$$

The $g_{2}\left(x, Q^{2}\right)$ structure function is related to the integral over this two-point green function. We don't know the exact relation between them, but at least we can say that it has two terms: the first one is a function of $Q^{2}$ and the second one is a Dirac Delta function which implies conservation of energy-momentum. To establish this relation, we resort to the phenomenological arguments. Obviously, the simplest choice for $\bar{g}_{2}\left(x, Q^{2}\right)$ is

$$
\bar{g}_{2}^{\text {valon }}\left(z, Q^{2}\right)=f\left(Q^{2}\right) \delta(z-1)
$$

at initial scale of $Q_{0}^{2}$, the photon probe detects only three valence quarks inside the proton. Hence, we can assume that $f\left(Q^{2}\right) \rightarrow A$ and we have:

$$
\bar{g}_{2}^{\text {valon }}\left(z, Q_{0}^{2}\right)=A \delta(z-1)
$$

The constant A can be determined from experimental data. Our motivation for this value comes from the phenomenological consideration which is required us to choose the initial input densities as $\delta(z-1)$ at $Q_{0}^{2}$. This mathematical boundary condition means that the internal structure of the valon cannot be resolved at $Q_{0}^{2}$. At this scale of $Q_{0}^{2}$, the nucleon can be considered as a bound state of three valence quarks that carry all the momentum and the spin of the nucleon. As $Q^{2}$ is increased, other partons can be resolved at the nucleon. 


\section{References}

[1] E. Shuryak, A.Vainshtein, Nucl. Phys. B 201, 141 (1982)

[2] E. Shuryak, A.Vainshtein, Phys.Lett. B105 (1981) 65-67

[3] Abe et al. (E143 Colaboration), Phys. Rev. D 58, 112003 (1998)

[4] Anthony et al. (E155 Collaboration), Phys. Lett. B 458, 529535 (1999); Phys. Lett. B 553, $18(2003)$

[5] Peter E. Bosted, (E155x Collaboration), RIKEN Review No. 28 (2000)

[6] V. A. Korotkov (HERMES Collaboration), PoS DIS 2010: 234, (2010)

[7] A. Airapetian et al. Eur. Phys. J. C 72, 1921 (2012)

[8] R. C. Hwa, Phys. Rev. D 22, 759 (1980)

[9] R. C. Hwa and C. B. Yang, Phys. Rev. C 66, 025205 (2002)

[10] G. Altarelli, S. Petrarca and F. Rapuano, Phys. Lett. B 373, (1996) 200 [hep$\mathrm{ph} / 9510346]$.

[11] F. Arash and A. N. Khorramian, Phys. Rev. C 67, 045201 (2003) [hep-ph/0303031].

[12] F. Arash, Phys. Lett. B 557, 38 (2003) [hep-ph/0301260].

[13] F. Arash, Phys. Rev. D 69, 054024 (2004) [hep-ph/0307221].

[14] F. Arash, Phys. Rev. D 50, 1946 (1994).

[15] F. Arash, Phys. Rev. D 52, 68-71 (1995).

[16] F. Arash, F. Taghavi Shahri, JHEP 07, 071 (2007)

[17] F. Arash, F.Taghavi Shahri, Phys. Lett. B 668, 193(2008) 
[18] F. Taghavi Shahri, F. Arash, Phys. Rev. C 82, 035205 (2010)

[19] A. Shahveh, F. Taghavi Shahri, F. Arash, Phys. Lett B 691, 32-36 (2010)

[20] M. G. Alekseev (COMPASS Collaboration), Phys. Lett. B 690, 466 (2010)

[21] A. Airapetian et al. Phys. Rev. D 75, 012007 (2007) [hep-ex/0609039]

[22] V. Yu. Alexakhin et al. (COMPASS Collaboration), Phys. Lett. B 647, 8-17 (2007) [hep-ex/0609038]

[23] K. Kramer,et al. Phys. Rev. Lett 95, 142002 (2005) [nucl-ex/0506005]

[24] K. Abe, et al. Phys. Lett. B 405, 180-190 (1997); Phys. Rev. Lett. 7926 (1997)

[25] K. Abe, et al. (E143 Collaboration), Phys. Rev. Lett 75, 25 (1995); Phys. Rev. Lett. 78, $815(1997)$

[26] J. L. Cortes, B. Pire, J. P. Ralston, Z. Phys. C 55, 409 (1992)

[27] K. Slifer et al. (RSS Collaboration), Phys. Rev. Lett 105, 101601 (2010)

[28] S. Wandzura, F. Wilczek, Phys. Lett. B 72, 195 (1977)

[29] X. Song, Phys. Rev. D 54, 1955 (1996)

[30] X. Song, Phys. Rev. D 63, 094019 (2001)

[31] A. Ali, V. M. Brauun, G.Hiller, Phys. Lett. B 266, 117 (1991)

[32] M. Wakamatsu, Phys. Lett. B 487, 118-124 (2000)

[33] Z. Alizadeh Yazdi, F. Taghavi-Shahri, F. Arash and M. E. Zomorrodian, Phys. Rev. C 89, $055201(2014)$

[34] M. Anselmino, M. Boglione, U. D'Alesio, A. Kotzinian, S. Melis, F. Murgia and A. Prokudin, Nucl. Phys. Proc. Suppl. 191 (2009) 98-107, [hep-ph/0812.4366] 
[35] V. Barone, F. Bradamantec, A. Martin, Progress in Particle and Nuclear Physics 65 (2010) 267-333

[36] F. R. P. Bissey, A. W. Thomas, I. R. Afnan, Phys. Rev. C 64, 024004 (2001); F. R. P. Bissey, V. A. Guzey, M. Strikman, A.W. Thomas, Phys. Rev. C 65, 064317 (2002); I. R. Afnan, et al., Phys. Rev. C 68, 035201 (2003)

[37] M. M. Yazdanpanah, A. Mirjalili, S. Atashbar Tehrani, F. Taghavi Shahri, Nucl. Phys. A 831, 243-262 (2009)

[38] Anthony et al. (E142 Collaboration), Phys. Rev. D 54, 6620 (1996)

[39] X. Zheng et al. (The Jefferson Lab Hall A Collaboration ), Phys. Rev. C 70, 065207 (2004)

[40] M. Posik et al. (The Jefferson Lab Hall A Collaboration ), Phys. Rev. Lett 113, $022002(2014)$

[41] H. Burkhardt and W. N. Cottingham, Ann. Phys. 56, 453 (1970)

[42] X. Ji, P. Unrau, Phys. Lett. B 333, 228 (1994)

[43] E. Stein, Phys. Lett. B 343, 369 (1995)

[44] I. Balitsky, V. Braun, A. Klesnichenko, Phys. Lett. B 242, 1990

[45] M. Gockeler et al. Phys. Rev. D 53, 2317 (1996)

[46] M. Osipko et al. Phys. Rev. D 71, 054007 (2005)

[47] Stefan pokorski," Gauge field theories ", Cambridge University press, (2000) 


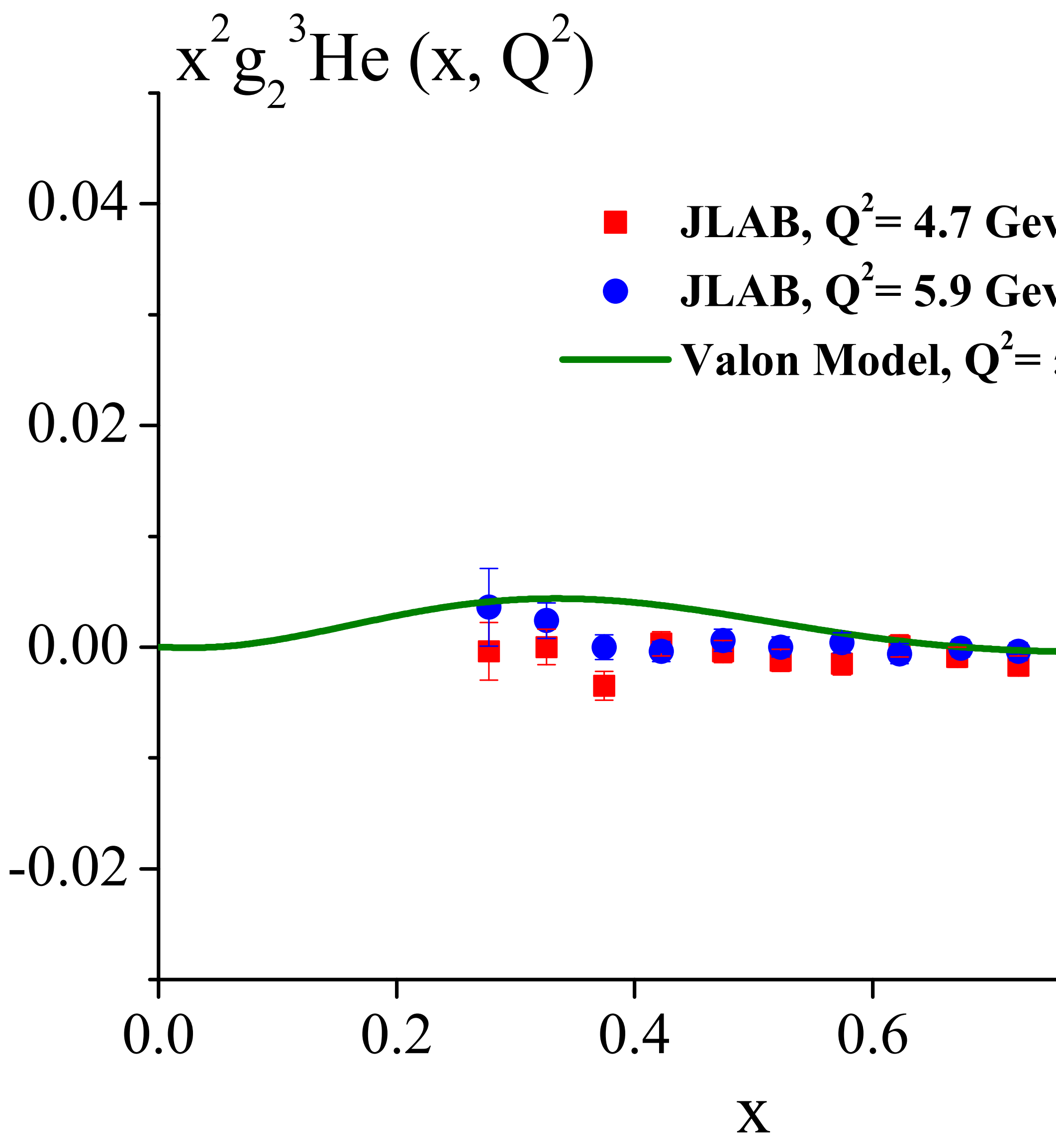

